\title{
Practical Aspects of SAT Solving
}

\author{
Armin Biere \\ Johannes-Kepler-Universität Linz, Austria
}

\begin{abstract}
SAT solving techniques are used in many automated reasoning engines. This talk gives an overview on recent developments in practical aspects of SAT solver development. Beside improvements of the basic conflict driven clause learning (CDCL) algorithm, we also discuss improving and integrating advanced preprocessing techniques as inprocessing during search. The talk concludes with a brief overview on current trends in parallelizing SAT.
\end{abstract}

